\title{
Performance Evaluation of Low-Cost BTEX Sensors and Devices within the EURAMET Key-VOCs Project $^{+}$
}

\author{
Laurent Spinelle ${ }^{1}$, Michel Gerboles ${ }^{1, *}$, Gertjan Kok ${ }^{2}$, Stefan Persijn ${ }^{2}$ and Tilman Sauerwald ${ }^{3}$ \\ 1 European Commission-Joint Research Centre, I-212027 Ispra (VA), Italy; laurent.spinelle@ec.europa.eu \\ 2 VSL Dutch Metrology Institute, 2629 JA Delft, The Netherlands; GKok@vsl.nl (G.K.); SPersijn@vsl.nl (S.P.) \\ 3 Laboratory for Measurement Technology, Universitaet des Saarlandes, Saarbruecken Campus A5 1, 66123 \\ Saarbruecken, Germany; t.sauerwald@lmt.uni-saarland.de \\ * Correspondence: michel.gerboles@ec.europa.eu; Tel.: +39-332-78-5652 \\ † Presented at the Eurosensors 2017 Conference, Paris, France, 3-6 September 2017.
}

Published: 29 August 2017

\begin{abstract}
The KEY-VOCs project is a EURAMET joint research project focused on key Volatile Organic Compounds (VOCs) in air. One of its activities is the evaluation of sensors-based measurement systems. In Europe, the monitoring of benzene in ambient air is mandatory as set by the European Directive for air quality (AQD) [1]. This Directive states that the reference method of measurement shall consist of active or on-line sampling followed by gas chromatography [2]. These methods are time consuming, expensive to implement and not easily portable prohibiting more local estimation of the population exposure. However, the AQD allows using indicative measurements with higher uncertainty than those of the reference methods. Sensor systems are good candidates for indicative methods with the additional ability of near-to real-time measurements.
\end{abstract}

Keywords: PID based sensors; semiconductor and amperometric sensor; mini GC; portable on-line measuring devices; benzene; volatile organic compounds

\section{Introduction}

Low-cost sensors are attracting more and more interest, as they would be able to provide a cheaper way to monitor air quality on a larger scale. However, if the current sensor technology can be considered efficient enough for inorganic gases such as $\mathrm{CO}, \mathrm{CO}_{2}$ and to a lower scale $\mathrm{O}_{3}$ and $\mathrm{NO}_{2}$ [3], that is not the case for volatile organic compounds (VOCs) and in particular BTEX (Benzene, Toluene, Ethylbenzene and Xylene). In the most recent review of sensors for the measurements of VOCs in ambient air [4,5], the authors concluded that the current sensor technology is not yet able to measure the concentrations expected in ambient air and that they generally suffer from poor selectivity.

Using the MACPoll Protocol of evaluation and calibration of low-cost gas sensors [6] which targeted low-costs sensors for inorganic gaseous, we present the evaluation of low-cost sensors and sensor based devices for benzene measurements. Indeed, the AQD defines as mandatory the monitoring of benzene in ambient air. In particular, it allows the use of indicative measurement methods but it requires the use of a selected method to meet a defined Data Quality Objective (DQO) of $30 \%$. This DQO is defined as the relative expanded uncertainty of measurement and it shall be assessed in the region of the limit value (LV) of $5 \mu \mathrm{g} / \mathrm{m}^{3}$ for the annual mean.

\section{Materials}

Out of an extended review of the potential sensor candidate [5], a set of six commercially available sensors or research prototypes were selected. Table 1 gather the brand, the model and the 
technology of the commercially available devices with their declared limit of detection and the experimental coefficient of determination of the linear regression. We also included in the evaluation process three devices that are part of research project. The first one uses a multi MOx/semiconductor sensor operated in Temperature Operation Cycle (TCO). The second one also uses TCO but with a $\mathrm{SiC}-\mathrm{FET}$ sensor prototype. The last one is a miniaturized Gas Chromatograph based on PID sensor.

Table 1. List of commercial sensors and portable devices selected and coefficient of determination of the linear regression $\left(R^{2}\right)$.

\begin{tabular}{|c|c|c|c|c|}
\hline Manufacturer/Institute & Model & Principle & Limit of Detection (ppm) & $R^{2}$ \\
\hline Aeroqual & VM & Semiconductor & $\begin{array}{c}0.001 \\
\text { (resolution 0.0001) }\end{array}$ & 0.00815 \\
\hline Ion Science & Tiger Select & $\begin{array}{c}\text { Portable hand held } \\
\text { based on PID sensors }\end{array}$ & $\begin{array}{c}0.010 \\
\text { (resolution 0.001) }\end{array}$ & 0.8011 \\
\hline Mocon Baseline & $\begin{array}{c}\text { item 045-014 } \\
\text { (color Blue) }\end{array}$ & PID & 0.00025 & 0.9924 \\
\hline Alphasense & PID-AH & PID & 0.0005 & 0.89165 \\
\hline \multirow{2}{*}{ Membrapor } & ETO/M-10 & Electrochemical & resolution 0.05 & 0.9605 \\
\hline & $\mathrm{CH} 2 \mathrm{O} / \mathrm{M}-10$ & Electrochemical & resolution 0.01 & 0.4040 \\
\hline $3 S$ & Not available & $\mathrm{MOS}+\mathrm{TCO}$ & Not available & 0.8992 \\
\hline University of Linkoping & Not available & SiC-FET & Not available & 0.3615 \\
\hline CNR-IMM Bologna & $\operatorname{miniGC}$ & Portable GC + PID & miniGC & 0.9989 \\
\hline
\end{tabular}

In a second stage, the performances of these sensors were evaluated under controlled conditions in an exposure chamber developed by the JRC. More details on this exposure chamber are described elsewhere [7]. This exposure chamber uses PID closed loop (Proportional, Integral and Derivative) in order to ensure the stability of the gaseous mixtures. The measurement of the levels of inorganic species was carried out by traditional analysers. For the organics species, a PTR-MS (Proton Transfer Reaction Mass Spectrometry) has been integrated as input of the PID loop [8]. A GC-PID 955 from Syntech was used in parallel as a reference measurement method to ensure selectivity, accuracy and traceability of the concentration of BTEX. All parameters are automatically and independently set and controlled. Conversely to other exposure chambers, the reference values of all compounds are measured allowing the full traceability to national/international units when evaluating sensors [7].

\section{Methodology and Results}

An extensive experimental design covering the evaluation of metrological parameters such as the response time, the repeatability, the lack of fit of calibration with hysteresis and short-term and long-term drifts have been applied. Meteorological interferences were also evaluated, in particular the effect of temperature, relative humidity, pressure and wind velocity. Finally, the cross sensitivity was characterized for the following interfering gaseous compounds: ozone, nitrogen oxides, carbon monoxide, toluene, xylene and five low-weight alkanes. This study gives preliminary results regarding the evaluation of the response time, the hysteresis in concentration, the influence of temperature, relative humidity and organic interfering compounds.

We evaluate the response time of sensors, t90, considering the time needed by the sensor to reach $90 \%$ of the final stable value in both increasing and decreasing concentration. For this test, the set point of concentration was set to around $10 \mathrm{ppb}$ of benzene and temperature and humidity were kept stable. The first observation was that the major part of the sensors was not able to detect such a low level of benzene. However, one PID sensor and the TCO MOx device showed a response time within the same range of the PTR-MS with $t_{90}=6 \mathrm{~min}$. In this particular experiment, the miniGC prototype cannot be considered as it gives a measurement every $15 \mathrm{~min}$. However, the miniGC shows a response time equal to the reference GC-PID 955 with $t_{90}=35 \mathrm{~min}$.

The original calibration levels were included between 0 to $3 \mathrm{ppb}$ of benzene by steps of $0.5 \mathrm{ppb}$ in randomized order to take into account any possible hysteresis effects. Generally, calibration lines were found linear. Table 1 gives the coefficient of determination of the calibration lines. Except the 
semiconductor sensor and one electrochemical, all the sensors showed a high correlation with the reference measurements.

The effect of other organic compounds such as toluene, xylene, ethane, propane, butane and pentane was also evaluated in a 4 steps procedure: (1) the sensors were exposed to zero air; (2) they were then exposed to benzene alone; (3) the interferent was then injected and the concentration is either measured by the GC-PID 955 or estimated by dilution; (4) finally, 3 ppb benzene was added to the mixture, keeping the experimental conditions stables in order to ensure a stable level of interferent. The influence of each interfering compound was determined separately. The tests were carried out at $22{ }^{\circ} \mathrm{C}$ and $60 \%$ of relative humidity. Figure 1 gives an estimation of the sensor sensitivity compared to their response to $3 \mathrm{ppb}$ on benzene.

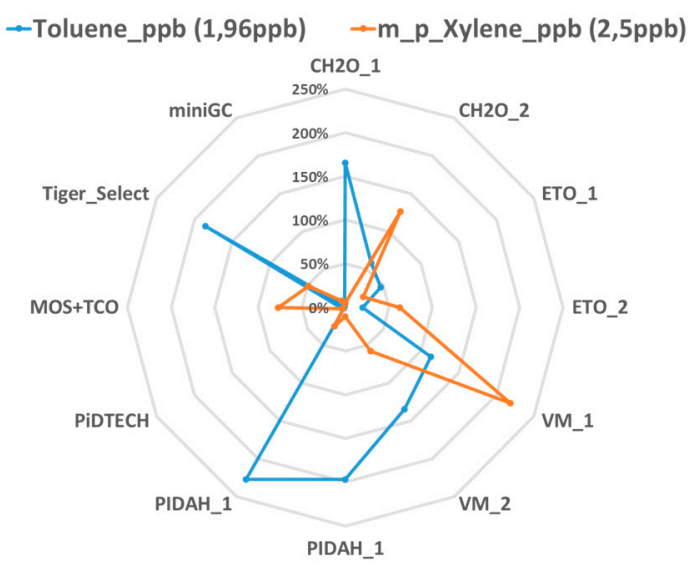

(a)

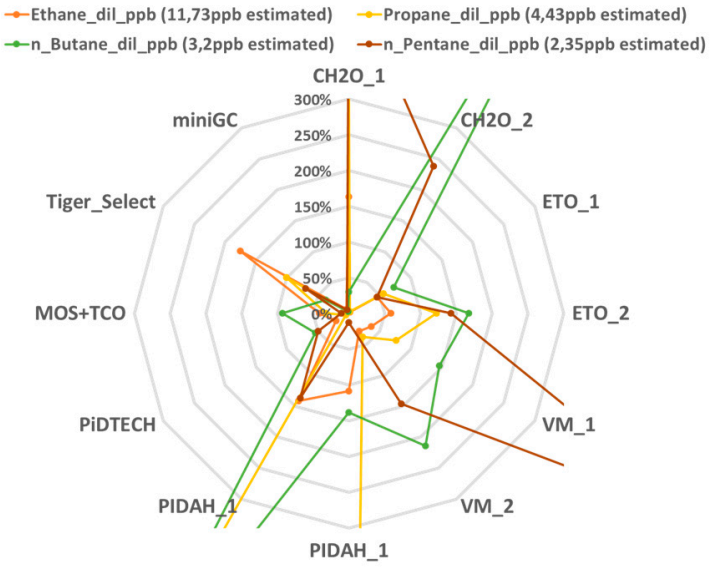

(b)

Figure 1. Estimation of the sensor sensitivity to organics interfering compounds.

As expected, the PID sensors (PID-AH and Tiger Select) showed the most important sensitivity to interfering compounds in terms of number of species. In particular they seem to be very sensitive to toluene, propane and butane. Both semiconductor sensors and the $\mathrm{CH} 2 \mathrm{O} / \mathrm{M}-10$ showed a crosssensitivity to alkane molecules and in a lower extend to toluene and xylene. A lower sensitivity was observed for the ETO/M-10 and the PiDTECH. The two devices which demonstrate the lowest interfering effect are the miniGC and the MOS + TCO.

The estimation of the dependence of sensors toward hysteresis was carried out using the calibration levels with a ramp of rising benzene followed with a ramp of decreasing levels and finally with another rising ramp. Three calibration lines were plotted, one for the 1 st ramp of rising levels, one for the falling levels and one for the 2nd ramp of rising levels. Only the PID sensors and electrochemical cells seems to be affected by hysteresis of concentration. However, this effect will have to be validated against the short term drift in a later stage.

The influence of the changes of temperature or relative humidity on the sensor's response were also studied. Two series of tests were conducted independently, generating ramps of temperature and humidity in a hysteresis cycle while gaseous levels in the chamber were kept constant. The ranges of temperature changed between 12 and $32{ }^{\circ} \mathrm{C}$ (by step of $5^{\circ} \mathrm{C}$ ) and the range of humidity was kept between $40 \%$ and $80 \%$ (by step of 10\%). In general all the tested sensors showed a strong dependency to temperature and relative humidity characterised respectively by linear and quadratic regression lines. Only the miniGC showed to be totally independent from any temperature and relative humidity effects. 


\section{Conclusions}

This laboratory evaluation shows that the current sensor technology is not able to accurately and selectively measure benzene at ambient levels. The PID sensors were generally found linear but they intrinsically suffer from high cross-sensitivity to other organic compounds. Amperometric and PID sensors seem also to suffer from huge hysteresis effect. Finally, both amperometric cells and semiconductor sensors suffer from a lack of sensitivity and high dependency to relative humidity and temperature. Only the sensor based devices (miniGC and MOS + TCO) were able to reach an interesting sensitivity associated with an almost non-existent cross sensitivity towards other gaseous interfering compounds. During this laboratory experiments, other parameters such as the repeatability, the limit of detection, the short term and long term drift have been estimated. Also, the influence of inorganic gas such as carbon monoxide $(\mathrm{CO})$, ozone $\left(\mathrm{O}_{3}\right)$ and nitrogen monoxide $(\mathrm{NO})$ and dioxide $\left(\mathrm{NO}_{2}\right)$ have been studied. These last results are still under evaluation. The results presented are only valid for the sensors' version under tests in the conditions and at the time of the tests.

Acknowledgments: This study was carried out within the EMRP Joint Research Project ENV56 KEY-VOCs. The EMRP is jointly funded by the EMRP participating countries within EURAMET and the European Union.

Conflicts of Interest: The authors declare no conflict of interest. The founding sponsors, EURAMET, had no role in the design of the study; in the collection, analyses, or interpretation of data; in the writing of the manuscript, and in the decision to publish the results.

\section{References}

1. Directive 2008/50/EC of the European Parliament and the Council of 21 May 2008 on ambient air quality and cleaner air for Europe. Available online: http://eur-lex.europa.eu/legal-content/EN/TXT/?uri= CELEX:32008L0050 (accessed on 7 August 2017)

2. Mooney, D. A guide for local authorities purchasing air quality monitoring equipment. Available online: https://clu-in.org/programs/21m2/lit_show.cfm?id=5587 (accessed on 7 August 2017)

3. Spinelle, L.; Gerboles, M.; Aleixandre, M. Performance evaluation of amperometric sensors for the monitoring of $\mathrm{O} 3$ and $\mathrm{NO} 2$ in ambient air at ppb level. Procedia Eng. 2015, 120, 480-483, doi:10.1016/j.proeng.2015.08.676.

4. Szulczyński, B.; Gębicki, J. Currently commercially available chemical sensors employed for detection of volatile organic compounds in outdoor and indoor air. Environments 2017, 4, 21, doi:10.3390/environments4010021.

5. Spinelle, L.; Gerboles, M.; Kok, G.; Persijn, S.; Sauerwald, T. Review of portable and low-cost sensors for the ambient air monitoring of benzene and other volatile organic compounds. Sensors 2017, 17, 1520, doi:10.3390/s17071520.

6. Spinelle, L.; Aleixandre, M.; Gerboles, M. Protocol of Evaluation and Calibration of Low-Cost Gas Sensors for the Monitoring of Air Pollution; Publications Office of the European Union: Luxembourg, 2013.

7. Spinelle, L.; Gerboles, M.; Aleixandre, M. Report of Laboratory and in-Situ Validation of Micro-Sensor for Monitoring Ambient Air-Ozone Micro-Sensors, $\alpha$ Sense, Model B4 O3 Sensors; Publications Office of the European Union: Luxembourg, 2013.

8. Spinelle, L.; Gerboles, M. Connection of a Rapid Online Measuring Device to the Atmosphere Test Control System; Publications Office of the European Union: Luxembourg, 2016.

(C) 2017 by the authors. Licensee MDPI, Basel, Switzerland. This article is an open access article distributed under the terms and conditions of the Creative Commons Attribution (CC BY) license (http://creativecommons.org/licenses/by/4.0/). 